\title{
Application of Attractor Selection to Adaptive Virtual Network Topology Control
}

\author{
Yuki Koizumi \\ Graduate School of \\ Information Science and \\ Technology, Osaka University \\ 1-5 Yamadaoka, Suita, Osaka \\ 565-0871, Japan \\ ykoizumi@ist.osaka- \\ u.ac.jp \\ Eiji Oki \\ NTT Network Service \\ Systems Laboratories \\ 3-9-11 Midori-cho, Musashino, \\ Tokyo 180-8585, Japan \\ oki@ice.uec.ac.jp
}

\author{
Takashi Miyamura \\ NTT Network Service \\ Systems Laboratories \\ 3-9-11 Midori-cho, Musashino, \\ Tokyo 180-8585, Japan \\ miyamura.takashi@lab. \\ ntt.co.jp
}

Kohei Shiomoto

NTT Network Service

Systems Laboratories

3-9-11 Midori-cho, Musashino,

Tokyo 180-8585, Japan

shiomoto.kohei@lab. ntt.co.jp

\author{
Shin'ichi Arakawa \\ Graduate School of \\ Information Science and \\ Technology, Osaka University \\ 1-5 Yamadaoka, Suita, Osaka \\ 565-0871, Japan \\ arakawa@ist.osaka- \\ u.ac.jp
}

Masayuki Murata

Graduate School of

Information Science and

Technology, Osaka University

1-5 Yamadaoka, Suita, Osaka

565-0871, Japan

murata@ist.osaka-u.ac.jp

\begin{abstract}
The growth of the Internet and emerging application layer technologies causes numerous changes in network environments. Therefore, it becomes important to achieve adaptive methods of controlling networks in addition to optimizing their performance. To achieve an adaptive network control method, we focus on attractor selection, which models behaviors where biological systems adapt to unknown changes in their surrounding environments and recover their conditions. In this paper, we show the applicability of the attractor selection to the adaptive virtual network topology (VNT) control in IP over wavelength-routed WDM networks. The simulation results indicate that our VNT control method based on attractor selection quickly and adaptively responds to various changes in traffic demand.
\end{abstract}

\section{Keywords}

Wavelength Division Multiplexing, Virtual Network Topology Control, Adaptability, Attractor Selection

\section{INTRODUCTION}

Wavelength Division Multiplexing (WDM) networks offer a flexible network infrastructure by using wavelength-routing capabilities. In such wavelength-routed WDM networks, a set of optical transport channels, called lightpaths, are established between nodes via optical cross-connects. Much research has been devoted to methods of carrying IP traffic, which is the majority of Internet traffic, over wavelength-routed WDM networks $[2,7,8,12,19]$. One ap-

Permission to make digital or hard copies of all or part of this work for personal or classroom use is granted without fee provided that copies are not made or distributed for profit or commercial advantage and that copies bear this notice and the full citation on the first page. To copy otherwise, to republish, to post on servers or to redistribute to lists, requires prior specific permission and/or a fee.

Bionetics '08, November 25-28, 2008, Hyogo, Japan

Copyright 2008 ICST 978-963-9799-35-6. proach to accommodating IP traffic on a wavelength-routed WDM network is to configure a virtual network topology (VNT), which consists of lightpaths and IP routers. To achieve effective transport of traffic, VNT control, which configures a VNT on the basis of given traffic demand matrices, has been investigated by many researchers $[14,17]$.

With the growth of the Internet, new application layer services, such as peer-to-peer networks, have emerged and these applications cause large fluctuations in network environments. For instance, the hugely fluctuating traffic demand caused by interactions between overlay networks and existing traffic engineering mechanisms has been revealed $[9,13]$. Therefore, it is important to achieve a VNT control method that is adaptive to changes in network environments.

The approaches to efficiently accommodating changing traffic demand on VNTs can basically be classified into two, i.e., offline and on-line approaches. In offline approaches, VNTs are statically constructed to efficiently accommodate one or multiple traffic demand matrices $[1,18]$. These approaches mainly assume that these traffic demand matrices will be available before the VNT is constructed. However, it is obvious that offline approaches cannot efficiently handle unexpected changes in traffic demand since VNTs are configured for a certain set of traffic demand matrices.

In contrast with offline approaches, on-line approaches dynamically reconfigure VNTs based on their detection of degraded performance or periodic measurements of the network status without a priori knowledge of future traffic demand $[4,16]$. Therefore, on-line approaches adapt to changes in traffic demand. We develop an online approach to achieve an adaptive VNT control method. In [16], a VNT reconfiguration method that uses given traffic demand matrices and configures an optimal VNT for the new traffic demand matrix was proposed. For the smooth transition of VNTs, this method designs a new VNT by solving linear programming with constraints on the number of changing lightpaths between the old and the new VNTs. In [4], the authors proposed an optimizationbased and heuristic VNT reconfiguration method based on periodic measurements of the load on lightpaths. This method adapts to changes in traffic demand by adding one new lightpath when con- 
gestion occurs on the VNT or deleting one underutilized lightpath on the assumption that traffic demand is changing gradually over long timescales.

Existing on-line VNT control methods assume that traffic demand is changing gradually and periodically as observed in [10]. However, if there are overlay networks on top of the network controlled by the VNT control mechanism, traffic demand fluctuates greatly and changes in traffic demand are unpredictable as has been pointed out $[9,13]$. Therefore, an important objective is to develop a VNT control method that adapts to various changes in traffic demand. To achieve this objective, we focus on mechanisms found in biological systems, which are adaptive against changes in their surrounding environments.

It is a well-known fact that mechanisms found in biological systems are robust and can handle changes in the environment [5]. Therefore, many methods that have been inspired by certain behaviors found in nature have been proposed. In this paper, we focus on attractor selection, which models behaviors where living organisms adapt to unknown changes in their surrounding environments and recover their conditions. In [6], the authors show an attractor selection model for E. coli cells to adapt to changes in the availability of a nutrient. As another model of an attractor selection, the mechanism for adaptability of a cell, which consists of a gene regulatory network and a metabolic network, is introduced in [3]. One successful proposal for adaptive network control based on attractor selection was presented in [11]. They proposed a path selection mechanism, which was robust against changes in the delay of paths, based on the attractor selection model introduced in [6]. The fundamental concept underlying attractor selection is that the system is driven by stochastic and deterministic behaviors, and these are controlled by simple feedback of current system conditions. This characteristic is one of the most important differences between attractor selection and other heuristic or optimization approaches. While existing heuristic approaches cannot handle unexpected changes in the environment, attractor selection has the capability of adapting to unknown changes since the system is driven by stochastic behavior and simple feedback of current system conditions. Therefore, we adopt attractor selection as the key mechanism in our VNT control method to attain adaptability against various changes in traffic demand.

In this paper, we propose an adaptive VNT control method based on the attractor selection described in [3]. We focus on the similarity of the layered architecture between this attractor selection model and the IP over WDM network. However, there are various differences that are mainly derived from the constraints of the physical network such as the number of transmitters and receivers. We need to interpret the attractor selection model for the VNT control method appropriately to develop an adaptive method based on attractor selection. We describe the interpretation of attractor selection for the VNT control method in more detail in Section 3.

The rest of this paper is organized as follows. Section 2 describes the attractor selection introduced in [3]. We then propose an adaptive VNT control method based on attractor selection in Section 3 and show its performance in Section 4. We conclude this paper in Section 5 .

\section{ATTRACTOR SELECTION}

Here, we briefly describe attractor selection, which is the key mechanism in our VNT control method. The original model for attractor selection was introduced in [3].

\subsection{Concept of Attractor Selection}

The dynamic system that is driven by attractor selection uses noise to adapt to environmental changes. In attractor selection, attractors are a part of the equilibrium points in the solution space in which the system conditions are preferable. The basic mechanism consists of two behaviors, i.e., deterministic and stochastic behaviors. When the current system conditions are suitable for the environment, i.e., the system state is close to one of the attractors, deterministic behavior drives the system to the attractor. Where the current system conditions are poor, stochastic behavior dominates over deterministic behavior. While stochastic behavior is dominant in controlling the system, the system state fluctuates randomly due to noise and the system searches for a new attractor. When the system conditions have recovered and the system state comes close to an attractor, deterministic behavior again controls the system. These two behaviors are controlled by simple feedback of the conditions in the system. In this way, attractor selection adapts to environmental changes by selecting attractors using stochastic behavior, deterministic behavior, and simple feedback. In the following section, we introduce attractor selection that models the behavior of gene regulatory and metabolic reaction networks in a cell.

\subsection{Cell Model}

The right of Fig. 1 is a schematic of the cell model used in [3]. It consists of two networks, i.e., the gene regulatory network in the box at the bottom of Fig. 1 and the metabolic reaction network in the box at the top.

Each gene in the gene regulatory network has an expression level of proteins and deterministic and stochastic behaviors in each gene control the expression level. Deterministic behavior controls the expression level due to the effects of activation and inhibition from the other genes. In Fig. 1, the effects of activation are indicated by the triangular-headed arrows and those of inhibition are indicated by the circular-headed arrows. In stochastic behavior, inherent noise randomly changes the expression level.

In the metabolic reaction network, metabolic reactions consume various substrates and produce new substrates. These metabolic reactions are catalyzed by proteins on corresponding genes. In the figure, metabolic reactions are illustrated as fluxes of substrates and catalyses of proteins are indicated by the dashed arrows. The changes in concentrations of metabolic substrates are given by metabolic reactions and the transportation of substrates from the outside of the cell. Some nutrient substrates are supplied from the environment by diffusion through the cell membrane.

The growth rate is determined by dynamics in the metabolic reactions. Some metabolic substrates are necessary for cellular growth, and thus the growth rate is determined as an increasing function of their concentrations. The gene regulatory network uses the growth rate as feedback of the conditions on the metabolic reaction network and controls deterministic and stochastic behaviors by using the growth rate. If the metabolic reaction network is in poor condition and the growth rate is small, the influence of stochastic behavior dominates deterministic behavior, triggering a search for a new attractor. During this phase, the expression levels are randomly changed by noise, and the gene regulatory network searches for a state that is suitable for the current environment. After the conditions of the metabolic reaction network have been recovered and the growth rate increases, deterministic behavior again drives the gene regulatory network to stable states.

The following section describes the mathematical model of attractor selection in more detail.

\subsection{Mathematical Model of Attractor Selec- tion}

The internal state of a cell is represented by a set of expression 


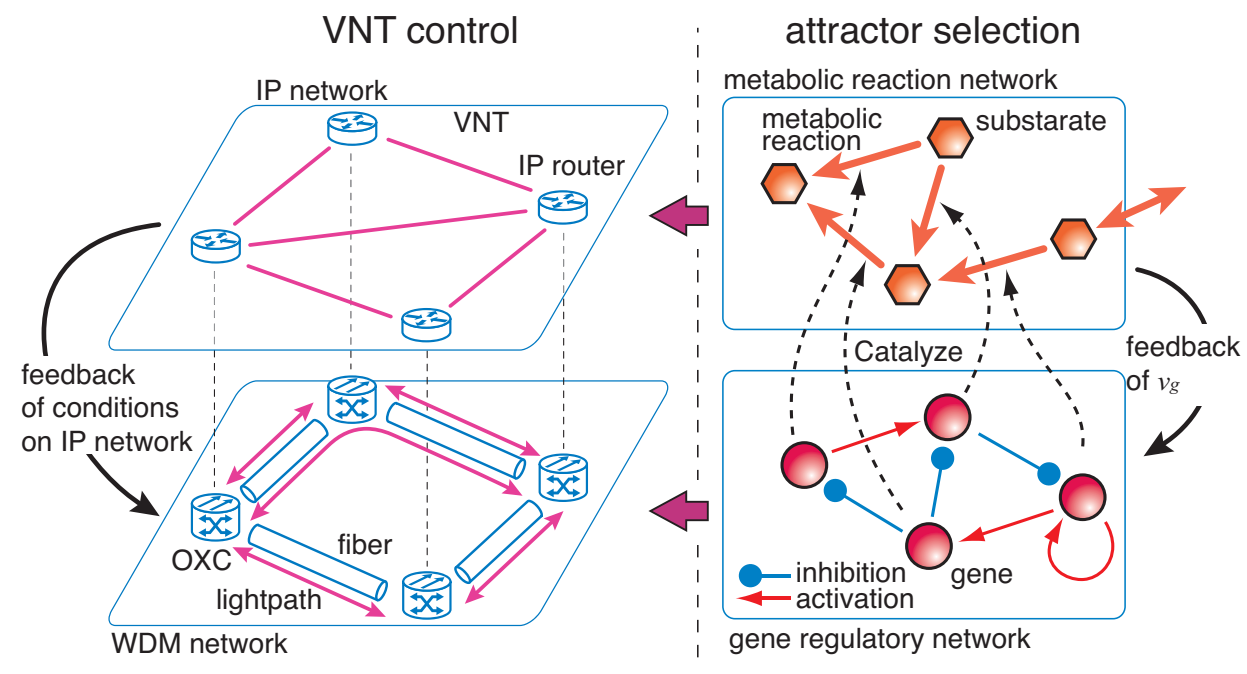

Figure 1: Interpretation of attractor selection into VNT control

levels of proteins on $n$ genes, $\left(x_{1}, x_{2}, \ldots, x_{n}\right)$, and concentrations of $m$ metabolic substrates, $\left(y_{1}, y_{2}, \ldots, y_{m}\right)$. The dynamics of the expression level of the protein on the $i$-th gene, $x_{i}$, is described as

$$
\frac{\mathrm{d} x_{i}}{\mathrm{~d} t}=\mathrm{f}\left(\sum_{j=1}^{n} W_{i j} x_{j}-\theta\right) \cdot v_{g}-x_{i} v_{g}+\eta .
$$

The first and second terms at the right hand side represent the deterministic behavior of gene $i$, and the third term represents stochastic behavior. In the first term, the regulation of protein expression levels on gene $i$ by other genes are indicated by regulatory matrix $W_{i j}$, which takes 1,0 , or -1 , corresponding to activation, no regulatory interaction, and inhibition of the $i$-th gene by the $j$-th gene. The rate of increase in the expression level is given by the sigmoidal regulation function, $\mathrm{f}(z)=1 /\left(1+e^{-\mu z}\right)$, where $z=\sum W_{i j} x_{j}-\theta$ is the total regulatory input with threshold $\theta$ for increasing $x_{i}$, and $\mu$ indicates the gain parameter of the sigmoid function. The second term represents the rate of decrease in the expression level on gene $i$. This term means that the expression level decreases depending on the current expression level. The last term at the right hand side in Eq. (1), $\eta$, represents molecular fluctuations, which is Gaussian white noise. Noise $\eta$ is independent of production and consumption terms and its amplitude is constant. The change in expression level $x_{i}$ is determined by deterministic behavior, the first and second terms in Eq. (1), and stochastic behavior $\eta$. The deterministic and stochastic behaviors are controlled by growth rate $v_{g}$, which represents the conditions of the metabolic reaction network.

In the metabolic reaction network, metabolic reactions, which are internal influences, and the transportation of substrates from the outside of the cell, which is an external influence, determine the changes in concentrations of metabolic substrates $y_{i}$. The metabolic reactions are catalyzed by proteins on corresponding genes. The expression level decides the strength of catalysis. A large expression level accelerates the metabolic reaction and a small expression level suppresses it. In other words, the gene regulatory network controls the metabolic reaction network through catalyses.

Several metabolic substrates are necessary for cellular growth. Growth rate $v_{g}$ is determined as an increasing function of the concentrations of these vital substrates. The gene regulatory network uses $v_{g}$ as the feedback of the conditions on the metabolic reaction network and controls deterministic and stochastic behaviors. If the concentrations of the required substrates decrease due to changes in the concentrations of nutrient substrates outside the cell, $v_{g}$ also decreases. By decreasing $v_{g}$, the effects that the first and second terms in Eq. (1) have on the dynamics of $x_{i}$ decrease, and the effects of $\eta$ increase relatively. Thus, $x_{i}$ fluctuates randomly and the gene regulatory network searches for a new attractor. The fluctuations in $x_{i}$ lead to changes in the rate of metabolic reactions via the catalyses of proteins. When the concentrations of the required substrates again increase, $v_{g}$ also increases. Then, the first and second terms in Eq. (1) again dominate the dynamics of $x_{i}$ over stochastic behavior, and the system converges to the state of the attractor. Since we mainly use the gene regulatory network and only mentioned concept of the metabolic reaction network, we have omitted a description of the metabolic reaction network from this paper. Readers can refer to [3] for a detailed description of the metabolic reaction network. The next section explains the VNT control method based on this attractor selection model.

\section{VNT CONTROL BASED ON ATTRACTOR SELECTION}

This section proposes a VNT control method based on the attractor selection model. We first introduce the network model that we use. Then, we outline our VNT control method based on attractor selection, and then describe our approach in detail.

\subsection{Network Model}

Our network consists of two layers: an optical and an IP layer as shown in the left of Fig. 1. On the optical layer, the WDM network consists of OXCs and optical fibers. VNT control configures lightpaths between IP routers via OXCs on the WDM network and these lightpaths and IP routers form a VNT. On the IP layer, packets are forwarded along the routes that are determined by IP routing on this VNT.

\subsection{Overview of VNT Control Based on At- tractor Selection}

In attractor selection, the gene regulatory network controls the metabolic reaction network, and the growth rate, which is the status of the metabolic reaction network, is recovered when the growth rate is degraded due to changes in the environment. In our VNT control method, the main objective is to recover the performance of 
the IP network by appropriately constructing VNTs when performance is degraded due to changes in traffic demand. Therefore, we interpret the gene regulatory network as a WDM network and the metabolic reaction network as an IP network, as shown in Fig. 1. The VNT control method drives the IP network in this way by constructing VNTs and the performance of the IP network recovers after it has degraded due to changes in traffic demand.

Our proposed approach works on the basis of periodic measurements of the link load, which is the volume of traffic on links, and it uses load information on links to know the conditions of the IP network. This information is converted to activity, which is the value to control deterministic and stochastic behaviors. We describe the activity in Section 3.3.3. Our method controls the deterministic and stochastic behaviors in the same way as attractor selection depending on the activity. We describe the deterministic and stochastic behaviors of our VNT control method in Section 3.3.1. Our method constructs a new VNT according to the system state of attractor selection, and the constructed VNT is applied as the new infrastructure for the IP network. By flowing traffic demand on this new VNT, the load on links in the IP network is changed, and our method again retrieves this information to know the conditions of the IP network.

\subsection{VNT Control Method Based on Attractor Selection}

This section describes our VNT control method in detail. The following sections use $i, j, s$, and $d$ as indexes of nodes, and $p_{i j}$ as an index of the source-destination pair from node $i$ to $j$.

\subsubsection{Dynamics of VNT Control}

We place genes on every source-destination pair in the WDM network and the expression level of each gene determines the number of lightpaths on those node pairs. To avoid confusion, we refer to genes placed on the WDM network as control units and the expression levels of the control units as control values.

The dynamics of $x_{p_{i j}}$ is defined by the following differential equation,

$$
\frac{\mathrm{d} x_{p_{i j}}}{\mathrm{~d} t}=v_{g} \cdot \mathrm{f}\left(\sum_{p_{s d}} W\left(p_{i j}, p_{s d}\right) \cdot x_{p_{s d}}-\theta_{p_{i j}}\right)-v_{g} \cdot x_{p_{i j}}+\eta,
$$

where $\eta$ represents white Gaussian noise, $\mathrm{f}(z)=1 /(1+\exp (-z))$ is the sigmoidal regulation function, and $v_{g}$ is the value that indicates the condition of the IP network. We use the same formula as in Eq. (1) to determine the control values. According to the observation in [3], we use white Gaussian noise with a mean of 0 and a variance of 0.1 for $\eta$.

The number of lightpaths between node pair $p_{i j}$ is determined according to value $x_{p_{i j}}$. We assign more lightpaths to a node pair that has a high control value than a node pair that has a low control value. Function $\mathrm{f}\left(z_{p_{i j}}-\theta_{p_{i j}}\right)$, where $z_{p_{i j}}=\sum_{p_{s d}} W\left(p_{i j}, p_{s d}\right) \cdot x_{p_{s d}}$, has its center at $z_{p_{i j}}=\theta_{p_{i} j}$ and exhibits rapid growth near $\theta_{p_{i j}}$. With smaller $\theta_{p_{i j}}$, the curve of $\mathrm{f}\left(z_{p_{i j}}-\theta_{p_{i j}}\right)$ is shifted in the negative direction, and therefore $\mathrm{f}\left(z_{p_{i j}}-\theta_{p_{i j}}\right)$ increases. This increases $\mathrm{d} x_{p_{i j}} / \mathrm{d} t$, and this then leads to an increase in $x_{p_{i j}}$. This is equivalent to increasing the number of lightpaths between $p_{i j}$ in our VNT control method. In the same way, a larger $\theta_{p_{i j}}$ leads to a decrease in $x_{p_{i j}}$ and then decreases the number of lightpaths between $p_{i j}$. Therefore, in our VNT control method, we control the number of lightpaths by adjusting $\theta_{p_{i j}}$ depending on the load on the link. Let $y_{p_{i j}}$ denote the load on the link between node pair $p_{i j}$. To assign more lightpaths to a node pair that has a highly loaded link, we decrease $\theta_{p_{i j}}$ for node pair $p_{i j}$ that has high $y_{p_{i j}}$. We determine $\theta_{p_{i j}}$ by using $\theta_{p_{i j}}=-\left(y_{p_{i j}}-y_{\min }\right) /\left(y_{\max }-y_{\min }\right) \times 2 \theta^{\star}-\theta^{\star}$, where $\theta^{\star}$ is the constant value that represents the range of $\theta_{p_{i j}}$, and $y_{\max }$ and $y_{\min }$ correspond to the maximum and minimum load in the network. If node pair $p_{i j}$ has no links, we use $y_{\min }$ as $y_{p_{i j}}$ to gradually modify the VNT.

\subsubsection{Regulatory Matrix}

The regulatory matrix is an important parameter since the deterministic behavior of our VNT control method is dominated by this matrix. Each element in the regulatory matrix, which is denoted as $W\left(p_{i j}, p_{s d}\right)$, represents the relation between node pair $p_{i j}$ and $p_{s d}$. The value of $W\left(p_{i j}, p_{s d}\right)$ is a positive number $\alpha_{A}$, zero, or a negative number $\alpha_{I}$, corresponding to activation, no relation, and inhibition of the control unit on $p_{i j}$ by the control unit on $p_{s d}$. If the control unit on $p_{i j}$ is activated by that on $p_{s d}$, increasing $x_{p_{s d}}$ leads to increasing $p_{i j}$. That is, node pair $p_{s d}$ increases the number of lightpaths on $p_{i j}$ in our VNT control method.

Let us consider three motivations for setting up or tearing down lightpaths for defining the regulatory matrix, i.e., establishing lightpaths for detouring traffic, increasing the number of lightpaths for the effective transport of traffic on the IP network, and decreasing the number of lightpaths due to a certain fiber being shared with other node pairs. First, for detouring traffic on the route from node $i$ to $j$ to other lightpaths, new lightpaths should be set up between node pair $p_{i j}$. Therefore, we interpret this motivation as the activation of the control unit on $p_{i j}$ by the control units on each node pair along the route of the lightpath between $p_{i j}$. Let us next consider the situation where a path on the IP network uses the lightpaths on $p_{i j}$ and $p_{s d}$. In this case, a certain amount of traffic on $p_{i j}$ is also transported on $p_{s d}$. Thus, if the number of lightpaths on $p_{i j}$ is increased, the number of lightpaths on $p_{s d}$ should also be increased for IP traffic to be effectively transported. Therefore, the control units on $p_{i j}$ and $p_{s d}$ activate each other. Finally, let us consider the relation between node pairs that share a certain fiber. Here, if the number of lightpaths on one node pair increases, the number of lightpaths on the other node pairs should decrease because of limitations on wavelengths. Therefore, the control unit on $p_{i j}$ is inhibited by the control unit on $p_{s d}$ if lightpaths between these node pairs share the same fiber. To achieve a more effective VNT control method in terms of optimal performance, other motivations such as the relation between adjacent node pairs should be considered. Since the main purpose in this research is to achieve an adaptive VNT control method, we consider these three motivations mentioned above.

The constant values, $\alpha_{A}$ and $\alpha_{I}$, represent the strength of activation and inhibition. The total regulatory input to each control unit, $z_{p_{i j}}=\sum_{p_{s d}} W\left(p_{i j}, p_{s d}\right) x_{p_{s d}}$, is inherent in Eq. (2) and should be independent of the number of control units since the appropriate regulatory input is determined by the sigmoid function, $\mathrm{f}\left(z_{p_{i j}}\right)$. To achieve a VNT control method that flexibly adapts to various environmental changes, Eq. (2) must have a sufficient number of equilibrium points, which are potential attractors depending on the surrounding environments. In [3], the authors evaluated their attractor selection model under a scenario where the gene regulatory network had 36 genes and each gene was activated or inhibited by other genes with a probability of 0.03 . They demonstrated that the attractor selection model was extremely adaptable against environmental changes. We determine $\alpha_{A}$ and $\alpha_{I}$ on the basis of their results. Since $z_{p_{i j}}$ cannot be retrieved prior to calculating Eq. (2), we use $z_{p_{i j}}^{\prime A}=\sum_{p_{s d}} W^{A}\left(p_{i j}, p_{s d}\right)$, and $z_{p_{i j}}^{\prime I}=\sum_{p_{s d}} W^{I}\left(p_{i j}, p_{s d}\right)$, where $W^{A}\left(p_{i j}, p_{s d}\right)$ and $W^{I}\left(p_{i j}, p_{s d}\right)$ are the binary variables. The variable, $W^{A}\left(p_{i j}, p_{s d}\right)$ takes 1 if the control unit on $p_{i j}$ is activated by that on $p_{s d}$, and otherwise 0 . To obtain the relation of inhibition, $W^{I}\left(p_{i j}, p_{s d}\right)$ is defined in the same way as $W^{A}\left(p_{i j}, p_{s d}\right)$. The two metrics, $z_{p_{i j}}^{\prime A}$ and $z_{p_{i j}}^{\prime \prime}$, indicate the total amount of activation or in- 
hibition on the control unit, $p_{i j}$, from the other control units. Each gene in [3] had $z^{\prime A}=0.03 \times 36=1.08$ since each gene was activated from 36 genes, including itself, with a probability of 0.03 . In our VNT control method, each control unit has an average of $z_{p_{i j}}^{A}=$ $\left(\sum_{p_{i j}} \sum_{p_{s d}} \alpha_{A} W^{A}\left(p_{i j}, p_{s d}\right)\right) / N$, where $N$ is the number of control units. Thus, we define $\alpha_{A}$ as $\alpha_{A}=1.08 N / \sum_{p_{i j}} \sum_{p_{s d}} W^{A}\left(p_{i j}, p_{s d}\right)$. In the same way, $\alpha_{I}$ is defined as $\alpha_{I}=1.08 N / \sum_{p_{i j}} \sum_{p_{s d}} W^{I}\left(p_{i j}, p_{s d}\right)$.

\subsubsection{Activity}

The growth rate is the value that indicates the conditions of the metabolic reaction network, and the gene regulatory network seeks to optimize the growth rate. In our VNT control method, we use the maximum link utilization on the IP network as a metric that indicates the conditions of the IP network. To retrieve the maximum link utilization, we collect the traffic volume on all links and select their maximum values. This information is easily and directly retrieved by SNMP. To avoid confusion, we will refer to the growth rate defined in our VNT control method as activity after this. This activity must be an increasing function for the goodness of the conditions of the target system, i.e., the IP network in our case, as mentioned in Section 2. Therefore, we convert the maximum link utilization on the IP network, $u_{\max }$, into the activity, $v_{g}$, as

$$
v_{g}= \begin{cases}\frac{\gamma}{1+\exp \left(\delta \cdot\left(u_{\max }-\zeta\right)\right)} & \text { if } u_{\max } \geq \zeta \\ \frac{\gamma}{1+\exp \left(\delta / 5 \cdot\left(u_{\max }-\zeta\right)\right)} & \text { if } u_{\max }<\zeta\end{cases}
$$

where $\gamma$ is the parameter that scales $v_{g}$ and $\delta$ represents the gradient of this function. The constant number, $\zeta$, is the threshold for the activity. One example curve for this activity function is plotted in Fig. 2. If the maximum link utilization is more than threshold $\zeta$, the activity rapidly approaches 0 due to the poor conditions of the IP network. Then, the dynamics of our VNT control method is governed by noise and the search for a new attractor. Where the maximum link utilization is less than $\zeta$, we increase the activity slowly with decaying gain in the activity to improve the maximum link utilization. Since improving the maximum link utilization from a higher value has a greater impact on the IP network than that from a lower value, even if the degree of improvement is the same, we differentiate the gain of the activity as depending on the current maximum link utilization. Moreover, by retaining the incentive for improving maximum link utilization, our VNT control method continuously attempts to improve the conditions of the IP network. Parameter $\gamma$ is set to 100 , which is a sufficiently shown as the enough large value for the gene regulatory network to strongly converge attractors despite the existence of noise in [3]. We set the target maximum link utilization, $\zeta$, to 0.5 and the gradient, $\delta$, to 50 to achieve quick responses to changes in $u_{\max }$.

\subsubsection{VNT Construction}

The number of lightpaths between node pair $p_{i j}$ is calculated from $x_{p_{i j}}$. To simplify the model of our VNT control method, we assume that the number of wavelengths on optical fibers will be sufficient and the number of transmitters and receivers of optical signals will restrict the number of lightpaths between node pairs. Each node has $P_{R}$ receivers and $P_{T}$ transmitters. We assign transmitters and receivers to lightpaths between $p_{i j}$ based on $x_{p_{i j}}$ normalized by the total control values for all the node pairs that use the transmitters or the receivers on node $i$ or $j$. The number of lightpaths between $p_{i j}, G_{p_{i j}}$, is determined as

$$
G_{p_{i j}}=\min \left(\left\lfloor P_{R} \cdot \frac{x_{p_{i j}}}{\sum_{s} x_{p_{s j}}}\right\rfloor,\left\lfloor P_{T} \cdot \frac{x_{p_{i j}}}{\sum_{d} x_{p_{i d}}}\right\rfloor\right) .
$$

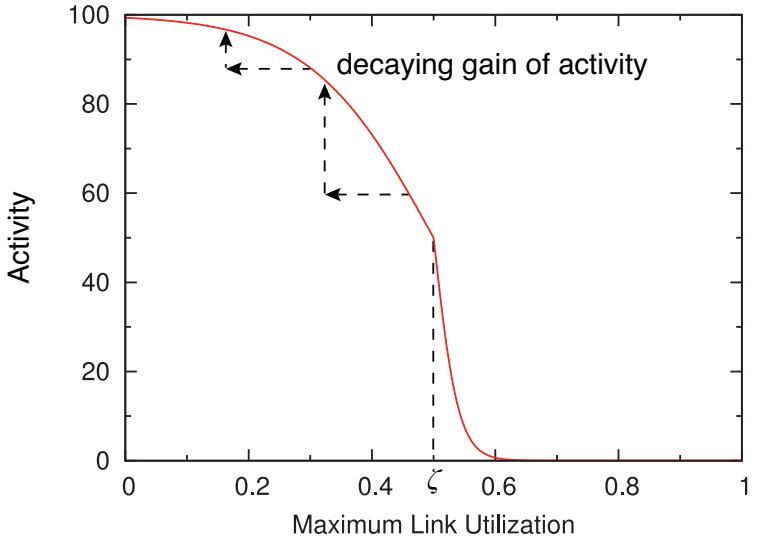

Figure 2: Activity

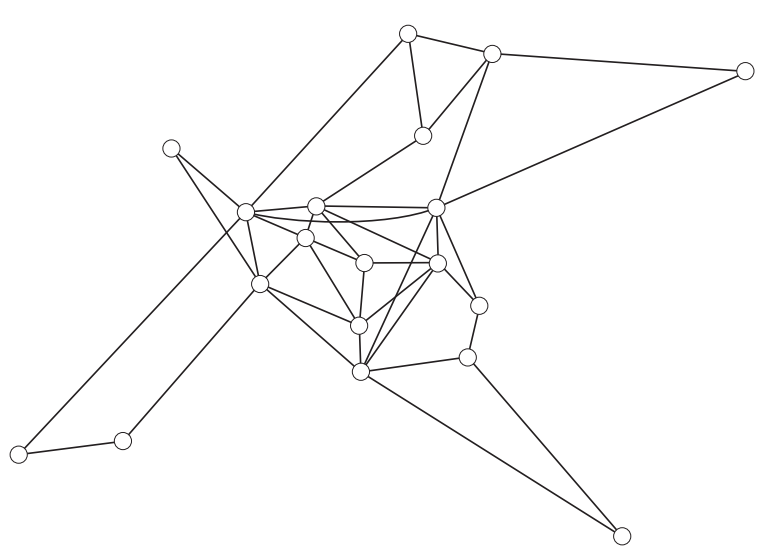

Figure 3: European Optical Network topology

Since we adopt the floor function for converting real numbers to integers, each node has residual transmitters and receivers. We assign one lightpath in descending order of $x_{p_{i j}}$ while the constraint on the number of transmitters and receivers is satisfied. Note that other constraints derived from physical resources such as the number of wavelengths on a fiber can easily be considered. For instance, restrictions on the number of wavelengths on a fiber are satisfied by adding $x_{p_{i j}}$ normalized by the total control values for all the node pairs that use the same fiber to Eq. (4).

\section{PERFORMANCE EVALUATION}

\subsection{Simulation Conditions}

We use the European Optical Network (EON) topology shown in Fig. 3 for the physical topology. The EON topology has 19 nodes and 39 bidirectional links. Each node has eight transmitters and eight receivers. We use randomly generated traffic demand matrices in the evaluations that followed.

We focus on changes in traffic demand in the IP network for the environmental changes. We consider two types of changes in traffic demand; the first included gradual and periodic changes and the second included sudden and sharp changes. By using Fourier series, traffic demand from node $i$ to $j$ at time $t, d_{i j}(t)$, changes gradually and periodically as

$$
d_{i j}(t)=\beta_{i j} \cdot\left(a+\sum_{h=1}^{H}\left(b_{i j}^{h} \cos \left(\frac{2 \pi t h}{T}\right)+c_{i j}^{h} \sin \left(\frac{2 \pi t h}{T}\right)\right),\right.
$$


where $T$ is the cycle of changes in traffic demand; we use 24 hours as a cycle in this simulation. The constant parameters $a, b_{i j}^{h}$, and $c_{i j}^{h}$ define the curve of $d_{i j}(t)$, and $\beta_{i j}$ scales $d_{i j}(t)$. Since our main objective is to achieve adaptability against changes in traffic demand and not to optimize the performance of the VNT control method for realistic traffic patterns, we simply generate the parameters as follows. Parameters $b_{i j}^{h}$ and $c_{i j}^{h}$ are uniformly distributed random numbers in a range from 0 to 1 . We set the constant value $a$ to $\sqrt{2}$ to ensure that $d_{i j}(t)$ is non-negative. The scale factor of traffic demand $\beta_{i j}$ follows a log-normal distribution with variance in the variable's logarithm, $\sigma^{2}$, according to the observation in [15]. We set $H$ to 1 . For sudden and abrupt changes in traffic demand, we randomly change $\beta_{i j}$ at certain intervals while keeping the expected value of total traffic demand in the network constant.

\subsection{Behaviors of VNT Control Based on At- tractor Selection}

This section explains the basic behaviors of our VNT control method. In the simulation experiments, we assume that our VNT control method will collect information about the load on links every 5 minutes.

We evaluate our VNT control method with the maximum link utilization in Fig. 4. The horizontal axis plots the time in hours and the vertical axis plots the maximum link utilization. The results for the first 24 hours have been omitted to disregard the transient phase during the simulation. Abrupt traffic changes occur every 3.6 hours and traffic demand continuously and gradually changes in the time between these abrupt traffic changes. Maximum link utilization degrades drastically every 3.6 hours due to the abrupt changes in traffic, but the maximum link utilization recovers shortly after this degradation.

To illustrate the adaptation mechanism of our VNT control method more clearly, we will present the control values, which determine the number of lightpaths between node pairs, and the activity, which is fed back to the our VNT control method and controls stochastic and deterministic behaviors, in Figs. 5 and 6, respectively. In Fig. 5, we selected ten control units out of 342 on all node pairs and have plotted the control values for these control units. When there are only periodic and gradual changes in traffic demand, our proposed method adjusts the control values depending on the changes in traffic demand. When maximum link utilization is degraded due to sharp changes in traffic demand, this degradation is reflected as a decrease in activity as shown in Figs. 4 and 6. As the result of the decreases in activity, stochastic behavior dominates over deterministic behavior in our VNT control method. This is observed as fluctuations in the control values in Fig. 5. Our method searches for a new VNT that is suitable for the changed traffic demand while stochastic behavior dominates deterministic behavior. After the new VNT is constructed and the maximum link utilization is recovered, activity increases, and then deterministic behavior again dominates in the VNT control method. In this way, our method adapts to both abrupt and gradual changes in traffic demand by controlling deterministic and stochastic behavior with activity.

\subsection{Adaptability to Changes in Traffic Demand}

We next evaluate the adaptability of our VNT control method to changes in traffic demand. For purposes of comparison, we use two existing heuristic VNT control methods. The MLDA (Minimum delay Logical topology Design Algorithm) [17] constructs VNTs on the basis of a given traffic demand matrix. The main objective of MLDA is to minimize the maximum link utilization. The basic idea behind MLDA is to place lightpaths between nodes in order of descending traffic demand. We use another VNT control method,

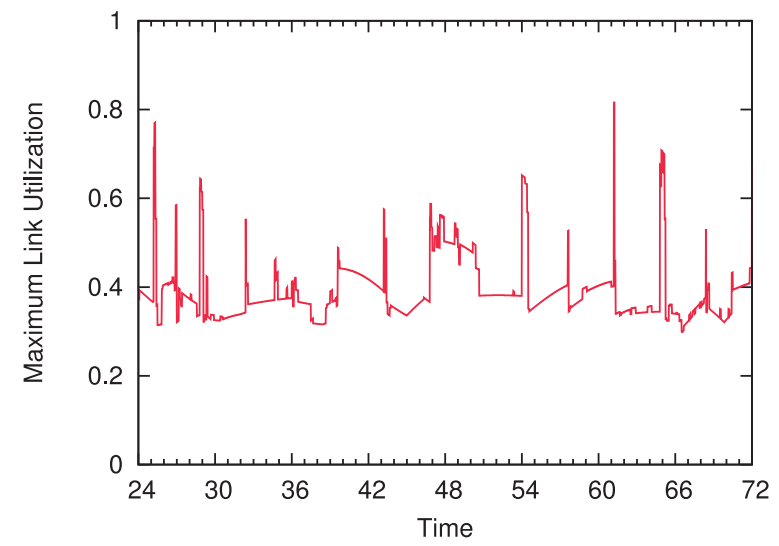

Figure 4: Maximum link utilization

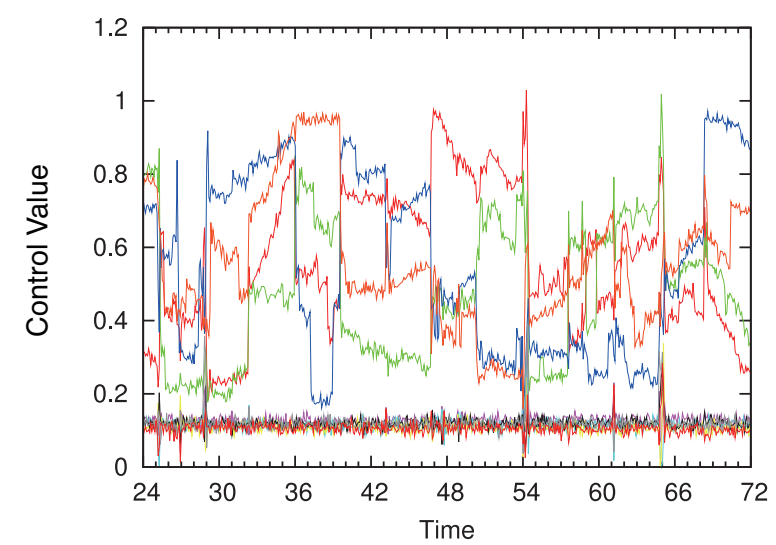

Figure 5: Control values

which is introduced in [4]. After this, we will refer to this VNT control method as "ADAPTATION." ADAPTATION aims at achieving adaptability against changes in traffic demand. This method reconfigures VNTs according to the load on links and the traffic demand matrix. ADAPTATION measures the actual load on links every 5 minutes and adds a new lightpath to the current VNT when congestion occurs. This method places a new lightpath on the node pair with the highest traffic demand among all node pairs that use the congested link. This decision is made according to the traffic demand matrix. However, to measure the traffic demand on all node pairs directly and in real-time is generally difficult due to the large overhead in collecting information. Therefore, we use the traffic demand matrix estimated with the method in [20] as the input parameter for ADAPTATION. In the simulation experiment, ADAPTATION reconfigures VNTs every 5 minutes using the measured load on links and the estimated traffic demand matrix. To simplify our evaluations, MLDA reconfigures VNTs every 60 minutes using the actual traffic demand matrix.

The maximum link utilization over time is shown in Fig. 7. The simulation conditions are the same as those discussed in Section 4.2. It is obvious that the maximum link utilization of MLDA continues to be high until the next reconfiguration is performed. Thus, degradation due to unsuitable VNT for changed traffic demand is retained for prolonged periods depending on the timing for the VNT reconfiguration. The recovery time, which is defined as the period until maximum link utilization is recovered, for our approach is much shorter than that for ADAPTATION, although both methods recon- 


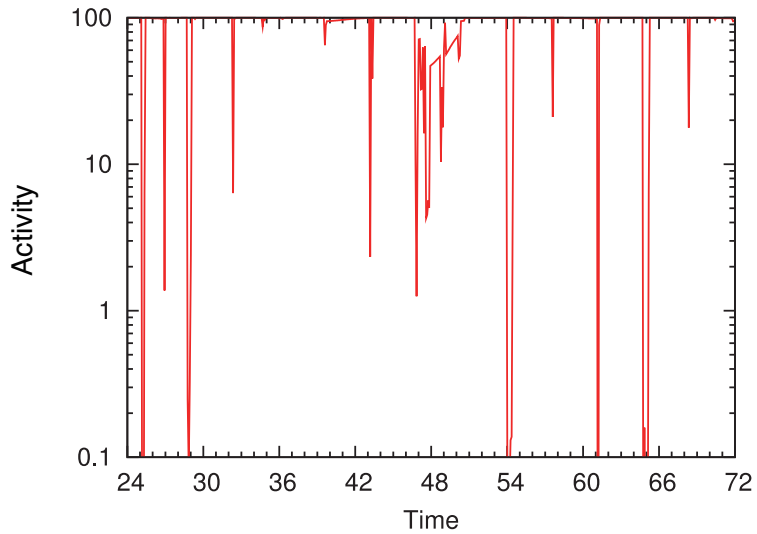

Figure 6: Activity

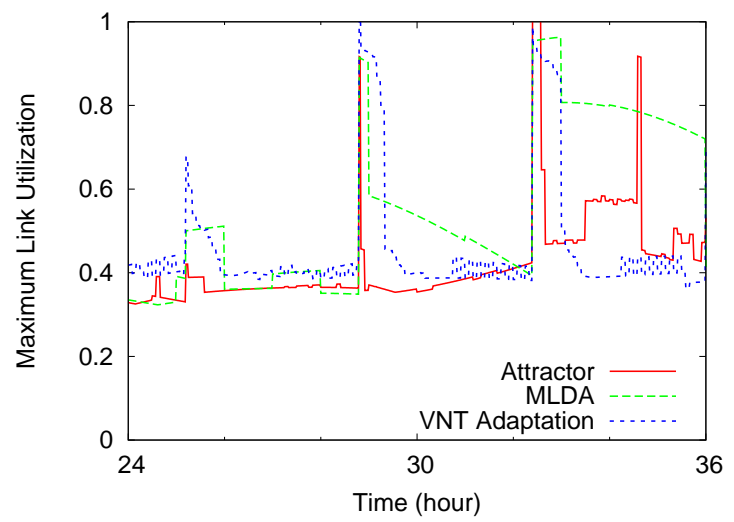

Figure 7: Adaptability of VNT control methods against changes in traffic demand

figure VNTs every 5 minutes. The recovery time for ADAPTATION is approximately the same as that for MLDA. Errors between estimated and actual traffic demand lead to incorrect decisions on selecting the node pair on which a new lightpath is placed. Thus, the efficiency of setting up lightpaths is degraded. In contrast with ADAPTATION, our method uses actual information, i.e., the measured load on links, and therefore quickly adapts to changes in traffic demand. A fixed amount of noise has a constant influence on our VNT control method even when the IP network has good conditions and activity is high. The effect of noise plays an important role in achieving adaptability against changes in traffic demand, as explained in Section 4.2. However, noise does not always have a beneficial effect on our proposed scheme due to its random nature. At time 35, the maximum link utilization with our VNT control method increases drastically even though abrupt changes in traffic demand do not occur. However, this degradation in maximum link utilization is immediately reflected as a decrease in activity, and maximum link utilization quickly recovers due to the adaptation mechanism described in Section 4.2. These results indicate that our method has capabilities for adapting to changes in traffic demand, and can adapt to these changes quickly.

\section{CONCLUSION}

We proposed a VNT control method that is adaptive to changes in traffic demand. It is based on attractor selection, which models the behaviors of biological systems that adapt to environmen- tal changes and recover their conditions. Our new approach is extremely adaptable to changes in traffic demand by appropriately controlling deterministic and stochastic behaviors depending on the activity, which is simple feedback of the conditions on the IP network. Our proposed method only uses load information on links to determine the activity. Since the load on links is directly retrieved within short intervals, our proposed method quickly and adaptively responds to changes in traffic demand. The simulation results indicated that our VNT control method quickly responds and adapts to changes in traffic demand. By using stochastic behavior and controlling it appropriately depending on the activity, our new approach adapts to various changes in traffic demand.

In our approach, stochastic behavior, i.e., noise, plays an important role in achieving adaptability against changes in traffic demand. In this paper, we defined the noise according to the observation in [3]. A future direction is to investigate a suitable noise amplitude for VNT control methods to achieve more efficient search for a new VNT.

\section{Acknowledghment}

This research was supported in part by "Special Coordination Funds for Promoting Science and Technology: Yuragi Project" of the Ministry of Education, Culture, Sports, Science and Technology, Japan and the National Institute of Information and Communications Technology of Japan (NICT). The first author received support from the JSPS Research Fellowships for Young Scientists.

\section{REFERENCES}

[1] G. Agrawal and D. Medhi. Lightpath topology configuration for wavelength-routed IP/MPLS networks for time-dependent traffic. In Proceedings of GLOBECOM, pages 1-5, Nov. 2006.

[2] J. Comellas, R. Martinez, J. Prat, V. Sales, and G. Junyent. Integrated IP/WDM routing in GMPLS-based optical networks. IEEE Network Magazine, 17(2):22-27, Mar./Apr. 2003.

[3] C. Furusawa and K. Kaneko. A generic mechanism for adaptive growth rate regulation. PLoS Computational Biology, 4(1):e3, Jan. 2008.

[4] A. Gençata and B. Mukherjee. Virtual-topology adaptation for WDM mesh networks under dynamic traffic. IEEE/ACM Transactions on Networking, 11(2):236-247, Apr. 2003.

[5] K. Kaneko. Life: An introduction to complex systems biology. Understanding Complex Systems. Springer, New York, 2006.

[6] A. Kashiwagi, I. Urabe, K. Kaneko, and T. Yomo. Adaptive response of a gene network to environmental changes by fitness-induced attractor selection. PLOS ONE, 1(1):e49, Dec. 2006.

[7] M. Kodialam and T. V. Lakshman. Integrated dynamic IP and wavelength routing in IP over WDM networks. In Proceedings of IEEE INFOCOM, pages 358-366, Apr. 2001.

[8] Y. Koizumi, S. Arakawa, and M. Murata. On the integration of IP routing and wavelength routing in IP over WDM networks. In Proceedings of SPIE, volume 6022, page 602205, 2005.

[9] Y. Koizumi, T. Miyamura, S. Arakawa, E. Oki, K. Shiomoto, and M. Murata. Stability of virtual network topology control for overlay routing services. Journal of Optical Networking, 7(7):704-719, July 2008.

[10] A. Lakhina, K. Papagiannaki, M. Crovella, C. Diot, E. D. Kolaczyk, and N. Taft. Structural analysis of network traffic 
flows. In Proceedings of ACM Sigmetrics, pages 61-72, June 2004.

[11] K. Leibnitz, N. Wakamiya, and M. Murata.

Biologically-inspired self-adaptive multi-path routing in overlay networks. Communications of the ACM, Special Issue on Self-Managed Systems and Services, 49(3):62-67, Mar. 2006.

[12] J. Li, G. Mohan, E. C. Tien, and K. C. Chua. Dynamic routing with inaccurate link state information in integrated IP over WDM networks. Computer Networks, 46:829-851, Dec. 2004

[13] Y. Liu, H. Zhang, W. Gong, and D. Towsley. On the interaction between overlay routing and underlay routing. In Proceedings of IEEE INFOCOM, pages 2543-2553, Mar. 2005.

[14] B. Mukherjee, D. Banerjee, S. Ramamurthy, and A. Mukherjee. Some principles for designing a wide-area WDM optical network. IEEE/ACM Transactions on Networking, 4(5):684-696, 1996.

[15] A. Nucci, A. Sridharan, and N. Taft. The problem of synthetically generating IP traffic matrices: initial recommendations. Computer Communication Review, 35(3):19-32, 2005.

[16] B. Ramamurthy and A. Ramakrishnan. Virtual topology reconfiguration of wavelength-routed optical WDM networks. In Proceedings of GLOBECOM, volume 2, pages 1269-1275, Nov. 2000.

[17] R. Ramaswami and K. N. Sivarajan. Design of logical topologies for wavelength-routed optical networks. IEEE Journal on Selected Areas in Communications, 14:840-851, June 1996.

[18] F. Ricciato, S. Salsano, A. Belmonte, and M. Listanti. Off-line configuration of a MPLS over WDM network under time-varying offered traffic. In Proceedings of IEEE INFOCOM, volume 1, pages 57-65, June 2002.

[19] T. Ye, Q. Zeng, Y. Su, L. Leng, W. Wei, Z. Zhang, W. Guo, and Y. Jin. On-line integrated routing in dynamic multifiber IP/WDM networks. IEEE Journal on Selected Areas in Communications, 22(9):1681-1691, Nov. 2004.

[20] Y. Zhang, M. Roughan, N. Duffield, and A. Greenberg. Fast accurate computation of large-scale IP traffic matrices from link loads. In Proceedings of ACM Sigmetrics, volume 31, pages 206-217, June 2003. 